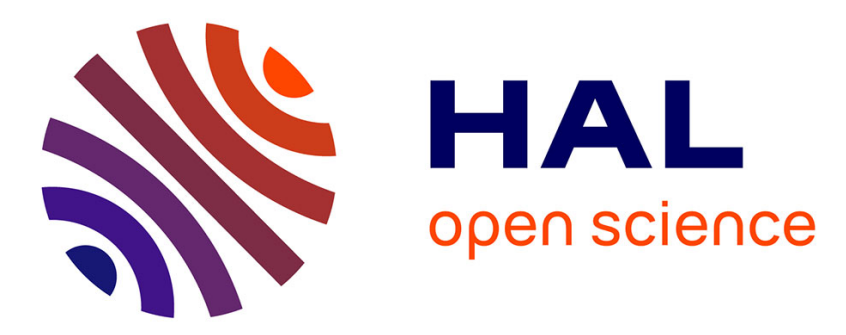

\title{
Influence of excitation pulse characteristics on resonant multiphoton ionization
}

\author{
M. Crance, S. Feneuille
}

\section{To cite this version:}

M. Crance, S. Feneuille. Influence of excitation pulse characteristics on resonant multiphoton ionization. Journal de Physique Lettres, 1976, 37 (12), pp.333-337. 10.1051/jphyslet:019760037012033300 . jpa-00231305

\section{HAL Id: jpa-00231305 https://hal.science/jpa-00231305}

Submitted on 1 Jan 1976

HAL is a multi-disciplinary open access archive for the deposit and dissemination of scientific research documents, whether they are published or not. The documents may come from teaching and research institutions in France or abroad, or from public or private research centers.
L'archive ouverte pluridisciplinaire HAL, est destinée au dépôt et à la diffusion de documents scientifiques de niveau recherche, publiés ou non, émanant des établissements d'enseignement et de recherche français ou étrangers, des laboratoires publics ou privés. 


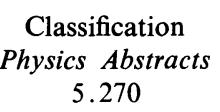

\title{
INFLUENCE OF EXCITATION PULSE CHARACTERISTICS ON RESONANT MULTIPHOTON IONIZATION
}

\author{
M. CRANCE and S. FENEUILLE \\ Laboratoire Aimé-Cotton, C.N.R.S. II, Université Paris-Sud \\ 91405 Orsay Cedex, France
}

(Reçu le 13 septembre 1976, révisé le 13 octobre 1976, accepté le 14 octobre 1976)

\begin{abstract}
Résumé. - L'objet de cette lettre est de montrer que, dans certains cas limites physiquement réalisables, un modèle simple, utilisant le formalisme de l'opérateur densité et une description classique du champ électromagnétique, permet d'étudier l'influence des caractéristiques (durée et forme) du pulse d'excitation sur l'ionisation multiphotonique résonnante.
\end{abstract}

Abstract. - The object of this letter is to show that, in some limiting cases, a simple model using an atomic density operator and a classical description of the field allows one to study the influence of the characteristics (duration and shape) of the excitation pulse on resonant multiphoton ionization.

During the last few years, mainly thanks to important advances in tunable laser technology, various phenomena which depend on light intensity (shift and asymmetry of resonances, variations of the nonlinearity) have been observed on resonant multiphoton ionization processes [1]. Some of them have been reproduced theoretically [2] by introducing higher order terms arising from the S-operator expansion within the framework of time-dependent perturbation theory. However, the corresponding calculations are extremely complicated even for the simplest atoms and, in any case, it is rather difficult from such numerical treatments to deduce general conclusions and to give a simple physical explanation of the observed phenomena.

This problem has recently been reconsidered from a quite different point of view by using a realistic model which can be solved exactly to give the ionization probability near a resonance as a function of time, light intensity and a few atomic parameters $[3,4,5]$. In particular, if higher order effects can be ignored both in the nonresonant process and in one of the two steps of the resonant process, the ionization cross-section and the non-linearity can be written, for continuous excitation, as analytical functions depending on two parameters only [3, 4]. Furthermore, the role of the light pulse duration has been emphasized in the general case [5]. However, this model utilizes a quantum description of the driving field ; and it is well known that this description is not convenient to characterize fields with an amplitude and/or a phase varying in time and thus, to take into account the influence of the excitation pulse shape, for example. The object of this letter is to show that, if higher order effects are important to the transition between the ground and the resonant states only, this difficulty can be avoided by using an atomic density operator and a classical description of the field. The solution is discussed in detail for square pulses and some indications are given for more realistic pulses.

Basically, we use the same model as in ref. $[3,5]$, where the validity conditions of the corresponding approximations are discussed in detail. However, one can recall that multiphoton ionization near a resonance is described in the following way :

i) a nonresonant $n$-photon process induced by an effective interaction $H_{n}^{\mathrm{E}}$ which is obtained from perturbation theory to $n$th order,

ii) a two-step process : first, a $p$-photon transition from the ground state $i$ to the quasiresonant state $\varphi$ (this first excitation is induced by an effective interaction $\left.H_{p}\right)$, then a $(n-p)$-photon transition from $\varphi$ to a continuum state $\Psi_{\mathrm{E}}$ (this second excitation is induced by an effective interaction $H_{n-p}^{\mathrm{E}}$ ).

For reasons of simplicity, all the states considered are assumed to be non-degenerate and only one continuum is introduced in this model. Under these conditions, if one uses a classical description of the driving field, the Hamiltonian takes a very compact form :

$$
H=H_{\mathrm{A}}+H_{\mathrm{AF}}
$$

where $H_{\mathrm{A}}$ is a reduced atomic Hamiltonian and $H_{\mathrm{AF}}$, 
an effective interaction between the atom and the field. $H_{\mathrm{A}}$ can be written :

$$
\begin{aligned}
H_{\mathrm{A}}=\hbar \Omega_{\mathrm{i}}|\mathrm{i}\rangle\left\langle\mathrm{i}\left|+\hbar \Omega_{\varphi}\right| \varphi\right\rangle\langle\varphi|+ \\
+\int_{I}^{\infty} E\left|\Psi_{\mathrm{E}}\right\rangle\left\langle\Psi_{\mathrm{E}}\right| \mathrm{d} E
\end{aligned}
$$

where $\hbar \Omega_{\mathrm{i}}$ and $\hbar \Omega_{\varphi}$ are the energies of the states i and $\varphi$, possibly light-shifted by nonresonant processes, $I$ is the ionization energy of the atom. If $\omega / 2 \pi, \varepsilon(t)$ and $\varphi(t)$ are respectively the frequency, the amplitude and the phase of the field, and if we keep only the energy conserving terms in the Hamiltonian (rotatingwave approximation), $H_{\mathrm{AF}}$ reduces to :

$$
\begin{aligned}
& H_{\mathrm{AF}}=|\varphi\rangle\langle\mathrm{i}|\left\langle\varphi\left|H_{p}\right| \mathrm{i}\right\rangle+ \\
&+\int_{I}^{\infty}\left|\Psi_{\mathrm{E}}\right\rangle\langle\varphi|\left\langle\Psi_{\mathrm{E}}\left|H_{n-p}^{\mathrm{E}}\right| \varphi\right\rangle \mathrm{d} E \\
&+\int_{I}^{\infty}\left|\Psi_{\mathrm{E}}\right\rangle\langle\mathrm{i}|\left\langle\Psi_{\mathrm{E}}\left|H_{n}^{\mathrm{E}}\right| \mathrm{i}\right\rangle \mathrm{d} E+\mathrm{C} . \mathrm{C} . \\
&\left\langle\varphi\left|H_{p}\right| \mathrm{i}\right\rangle=\hbar K \mathrm{e}^{-i p[\omega t+\varphi(t)]} \\
&=\hbar k \varepsilon^{p} \mathrm{e}^{-i p[\omega t+\varphi(t)]} \\
&\left\langle\Psi_{\mathrm{E}}\left|H_{n-p}^{\mathrm{E}}\right| \varphi\right\rangle=\hbar L_{\mathrm{E}} \mathrm{e}^{-i(n-p)[\omega t+\varphi(t)]} \\
&=\hbar l_{\mathrm{E}} \varepsilon^{n-p} \mathrm{e}^{-i(n-p)[\omega t+\varphi(t)]} \\
&\left\langle\Psi_{\mathrm{E}}\left|H_{n}^{\mathrm{E}}\right| \mathrm{i}\right\rangle=\hbar J_{\mathrm{E}} \mathrm{e}^{-i n[\omega t+\varphi(t)]} \\
&=\hbar j_{\mathrm{E}} \varepsilon^{n} \mathrm{e}^{-i n[\omega t+\varphi(t)]} ;
\end{aligned}
$$

$k, l_{\mathrm{E}}$, and $j_{\mathrm{E}}$ are atomic parameters and are assumed to be real.

Now, if the condition $2 p \leqslant n$ is satisfied, the $n$ and $(n-p)$-photon processes can be described, in general, by retaining the lowest-order non-vanishing terms and it is clear that atomic coherences within continuum states can be ignored. With this approximation, the atomic density operator can be restricted to :

$$
\begin{aligned}
\rho= & \rho_{\mathrm{ii}}|\mathrm{i}\rangle\left\langle\mathrm{i}\left|+\rho_{\varphi \varphi}\right| \varphi\right\rangle\langle\varphi|+ \\
& +\int_{I}^{\infty} \rho_{\mathrm{EE}}\left|\Psi_{\mathrm{E}}\right\rangle\left\langle\Psi_{\mathrm{E}}\right| \mathrm{d} E \\
& +\rho_{\mathrm{i} \varphi}|\mathrm{i}\rangle\left\langle\varphi\left|+\rho_{\varphi \mathrm{i}}\right| \varphi\right\rangle\langle\mathrm{i}| \\
& +\int_{I}^{\infty}\left\{\rho_{\mathrm{iE}}|\mathrm{i}\rangle\left\langle\Psi_{\mathrm{E}}\left|+\rho_{\mathrm{Ei}}\right| \Psi_{\mathrm{E}}\right\rangle\langle\mathrm{i}|\right\} \mathrm{d} E \\
& +\int_{I}^{\infty}\left\{\rho_{\varphi \mathrm{E}}|\varphi\rangle\left\langle\Psi_{\mathrm{E}}\left|+\rho_{\mathrm{E} \varphi}\right| \Psi_{\mathrm{E}}\right\rangle\langle\varphi|\right\} \mathrm{d} E,
\end{aligned}
$$

and, the equations of motion for the density matrix elements become :

$$
\begin{aligned}
\dot{\rho}_{\mathrm{ii}} & =-\dot{\rho}_{\varphi \varphi}=2 K b_{\varphi \mathrm{i}} \\
\dot{a}_{\varphi \mathrm{i}} & =2 \delta^{\prime} b_{\varphi \mathrm{i}} \\
\dot{b}_{\varphi \mathrm{i}} & =-2 \delta^{\prime} a_{\varphi \mathrm{i}}-K\left(\rho_{\mathrm{ii}}-\rho_{\varphi \varphi}\right) \\
\dot{\rho}_{\mathrm{EE}} & =-2 L_{\mathrm{E}} b_{\mathrm{E} \varphi}-2 J_{\mathrm{E}} b_{\mathrm{Ei}} \\
\dot{a}_{\mathrm{E} \varphi} & =2 \Delta_{\mathrm{E}} b_{\mathrm{E} \varphi}-K b_{\mathrm{Ei}}-J_{\mathrm{E}} b_{\varphi \mathrm{i}} \\
\dot{a}_{\mathrm{Ei}} & =2\left(\delta^{\prime}+\Delta_{\mathrm{E}}\right) b_{\mathrm{Ei}}-K b_{\mathrm{E} \varphi}+L_{\mathrm{E}} b_{\varphi \mathrm{i}} \\
\dot{b}_{\mathrm{E} \varphi} & =-2 \Delta_{\mathrm{E}} a_{\mathrm{E} \varphi}+K a_{\mathrm{Ei}}-J_{\mathrm{E}} a_{\varphi \mathrm{i}}-L_{\mathrm{E}} \rho_{\varphi \varphi} \\
\dot{b}_{\mathrm{Ei}} & =-2\left(\delta^{\prime}+\Delta_{\mathrm{E}}\right) a_{\mathrm{Ei}}+K a_{\mathrm{E} \varphi}-L_{\mathrm{E}} a_{\varphi \mathrm{i}}-J_{\mathrm{E}} \rho_{\mathrm{ii}}
\end{aligned}
$$

where :

$$
\begin{gathered}
a_{\varphi \mathrm{i}}+i b_{\varphi \mathrm{i}}=\rho_{\varphi \mathrm{i}} \mathrm{e}^{i p[\omega t+\varphi(t)]} \\
a_{\mathrm{E} \varphi}+i b_{\mathrm{E} \varphi}=\rho_{\mathrm{E} \varphi} \mathrm{e}^{i(n-p)[\omega t+\varphi(t)]} \\
a_{\mathrm{Ei}}+i b_{\mathrm{Ei}}=\rho_{\mathrm{Ei}} \mathrm{e}^{i n[\omega t+\varphi(t)]} . \\
\delta^{\prime}=\frac{1}{2}\left\{\Omega_{\varphi}-\Omega_{\mathrm{i}}-p[\omega+\dot{\varphi}(t)]\right\}=\delta-\frac{1}{2} p \dot{\varphi}(t) \\
\Delta_{\mathrm{E}}=\frac{1}{2}\left\{\hbar^{-1} E-\Omega_{\varphi}-(n-p)[\omega+\dot{\varphi}(t)]\right\} .
\end{gathered}
$$

If $P$ is the number of atoms interacting with the field, the total number of produced ions is given by :

$$
N=2 P \int_{-\infty}^{+\infty} \mathrm{d} t \int_{I}^{\infty} \mathrm{d} E\left\{L_{\mathrm{E}} b_{\mathrm{E} \varphi}+J_{\mathrm{E}} b_{\mathrm{Ei}}\right\},
$$

so long as $N / P$ remains much smaller than one.

Eqs. (5) are nothing more than the familiar Bloch equations for a two-level atom [6] and therefore, since the equations of motion separate completely for the two discrete states, the approximations made here are exactly the same as in ref. [3]. However, density matrix formalism and classical description of the field offer an important advantage : $K, J_{\mathrm{E}}$ and $L_{\mathrm{E}}$ are allowed to vary in time and, thus, the influence of the excitation pulse shape can be studied.

It has already been noted that, to obtain the total number of ions produced, the solutions of the equations of motion must be integrated over energy. In the general case, this integration would be very difficult to perform but one can assume that $J_{\mathrm{E}}$ and $L_{\mathrm{E}}$ do not vary rapidly with $E$ and, thus, one can consider them as energy independent over the whole range of integration. Moreover, if $n \omega+\Omega_{\mathrm{i}}-I$ is much larger than the mean value of $K, \bar{K}$, the integration range can be extended to $-\infty$ without introducing significant errors.

With these supplementary approximations, which are valid of course in most realistic cases, the solution corresponding to square pulse excitation :

$$
\begin{gathered}
K=\bar{K}, \quad J=\bar{J}, \quad L=\bar{L} \text { if } 0 \leqslant t \leqslant T \\
K=J=L=0 \text { for } t<0 \text { and } t>T
\end{gathered}
$$


can be obtained in a straightforward way and one obtains finally :

$$
\begin{aligned}
N & =N_{\mathrm{D}}\left\{\left[\frac{1}{2}+\frac{(\delta-\bar{K} / A)^{2}}{2\left(\bar{K}^{2}+\delta^{2}\right)}\right]+\right. \\
& \left.+\frac{\sin \left(\bar{K}^{2}+\delta^{2}\right)^{1 / 2} T}{\left(\bar{K}^{2}+\delta^{2}\right)^{1 / 2} T}\left[\frac{1}{2}-\frac{(\delta-\bar{K} / A)^{2}}{2\left(\bar{K}^{2}+\delta^{2}\right)}\right]\right\} \\
& =N_{\mathrm{D}}\left[1+\left\{1-\frac{\sin \bar{K} T\left(1+y^{2} / A^{2}\right)^{1 / 2}}{\bar{K} T\left(1+y^{2} / A^{2}\right)^{1 / 2}}\right\} \times\right. \\
& \left.\times\left\{\frac{1-A^{2}}{2\left(A^{2}+y^{2}\right)}-\frac{2 y}{2\left(A^{2}+y^{2}\right)}\right\}\right]
\end{aligned}
$$

where $N_{\mathrm{D}}=2 \pi P \bar{J}^{2} T$ is the number of ions which would be produced if the nonresonant process were the only one, $A=\bar{J} / \bar{L}$ and $y=\delta A / \bar{K}=\delta j / k l$. If the pulse area, $\theta=2 \bar{K} T$, is large, only the first term in eq. $(8 a)$ is important and one finds again the result given in ref. [3] for continuous excitation. The corresponding spectral response function, $N / N_{\mathrm{D}}$ is the sum of a constant (direct process), a lorentzian term (two-step process and corresponding alteration of direct process) and a dispersion term (interference between the two processes). The second term in eq. (8a) describes the transient part of the phenomenon and it clearly comes from Rabi nutation of the Bloch vector [6] corresponding to the two discrete levels. Its relative importance depends essentially on $\theta$ and if $\theta$ is larger than $3 \pi$, the relative difference between the values of $N, N_{\mathrm{C}}$ and $N_{\mathrm{P}}$, corresponding respectively to continuous and square-pulsed excitation, is smaller than $10 \%$ for any $\delta$ and any $A$. However, if we define $\theta_{\mathrm{s}}$ by the minimum value of $\theta$ for which the absolute difference between $\left(N_{\mathrm{C}} / N_{\mathrm{D}}\right)$ and $\left(N_{\mathrm{P}} / N_{\mathrm{D}}\right)$ becomes negligible (i.e. smaller than a few per cent) for any $\delta, \theta_{\mathrm{s}}$ depends on the value of $A$ (which varies as $\bar{\varepsilon}^{p}$ ) and more precisely decreases when $A$ increases. Some examples of such resonance profiles are given in figure 1 and a detailed analysis shows that for $\theta \ll \theta_{\mathrm{s}}$, the resonance peak becomes very weak and its position does not depend on the light

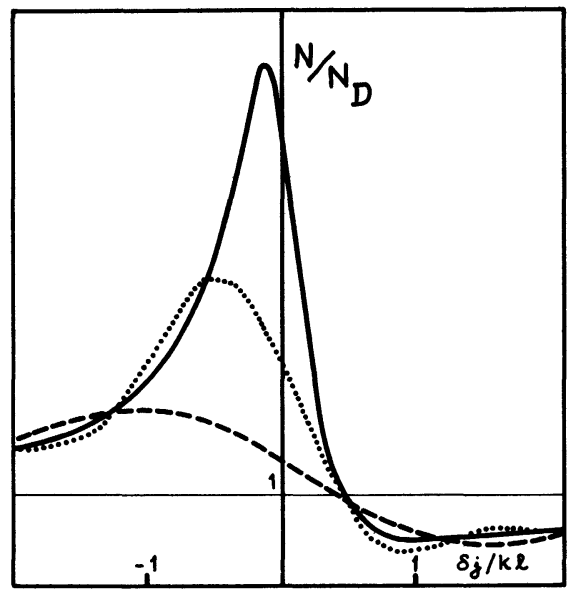

Fig. 1. - Square pulse excitation. Influence of the pulse area. $A^{2}=0.1 ;-\theta=\pi, \infty ; \ldots \theta=\pi / 2 ; \cdots \theta=\pi / 4$. intensity, while for continuous excitation $\left(\theta>\theta_{\mathrm{s}}\right)$, this position varies as $\bar{K} \bar{J} / \bar{L}$ which is proportional to $\bar{\varepsilon}^{2 p}$. Another way to describe transcient effects is to consider that, when $\theta$ decreases, the direct process is enhanced with respect to the two-step process and, actually, the resonance profile is not very different from the one which would be obtained with continuous excitation but, for an effective value of $A$ larger than the real one. For precise comparison with experimental data, care must be taken, however, since all the previous results are valid only if relaxation processes and in particular spontaneous emission can be ignored. If $\Gamma$ is a typical relaxation constant for the considered problem, this is true only if $\bar{K} \gg \Gamma$ and $\Gamma T \ll 1$. If $\Gamma T$ is much larger than one, the correct result is always the one derived for continuous excitation provided that $\bar{K} \gg \Gamma$. At this point, let us remark that another advantage of density matrix formalism is the possibility of introducing relaxation in a simple way, but the corresponding results are very complicated and it is useless to discuss them in this letter.

Now, to take complete advantage of the formalism introduced here, we have to discuss the influence of the pulse shape. For non-square pulses, it is no longer possible to find analytical solutions but one can perform numerical calculations for particular cases. Some examples of such calculations (carried out on the UNIVAC 1110 at Université Paris-Sud) are given in figures 2,3 and 4 . The corresponding pulse shapes are the following :

a) $K(t)=(2 e)^{1 / 2} K_{0}(t / \tau) \exp -(t / \tau)^{2}$ for $t \geqslant 0$

$$
\begin{array}{ll}
K(t)=0 & \text { for } t<0 \\
\dot{\varphi}(t)=0 &
\end{array}
$$

b) $K(t)=K_{0} \sin 2 \pi(t / 2 \tau)$ for $0 \leqslant t \leqslant \tau$

$$
\begin{array}{ll}
K(t)=0 & \text { for } t<0, \text { and } t>\tau \\
\dot{\varphi}(t)=0 &
\end{array}
$$

c) $K(t)=K_{0}[(t / \tau)-1]^{2}$ for $0 \leqslant t \leqslant \tau$

$$
\begin{array}{ll}
K(t)=0 & \text { for } t<0 \text { and } t>\tau \\
\dot{\varphi}(t) & =0
\end{array}
$$

and we have chosen $n=3, p=1$. If we denote by $J_{\mathrm{M}}$ and $L_{\mathrm{M}}$, the peak values of $J(t)$ and $L(t)$, it appears that the spectral response function depends essentially on the three following parameters : the area of the pulse

$$
\begin{gathered}
\theta=\int_{-\infty}^{+\infty} 2 K(t) \mathrm{d} t \\
A_{\mathrm{M}}^{2}=\left(J_{\mathrm{M}} / L_{\mathrm{M}}\right)^{2} \text { and } x^{2}=A^{2} / A_{\mathrm{M}}^{2},
\end{gathered}
$$

where $A^{2}$ is defined by :

$$
A^{2}=\int_{-\infty}^{+\infty} J(t)^{2} \mathrm{~d} t / \int_{-\infty}^{+\infty} L(t)^{2} \mathrm{~d} t
$$




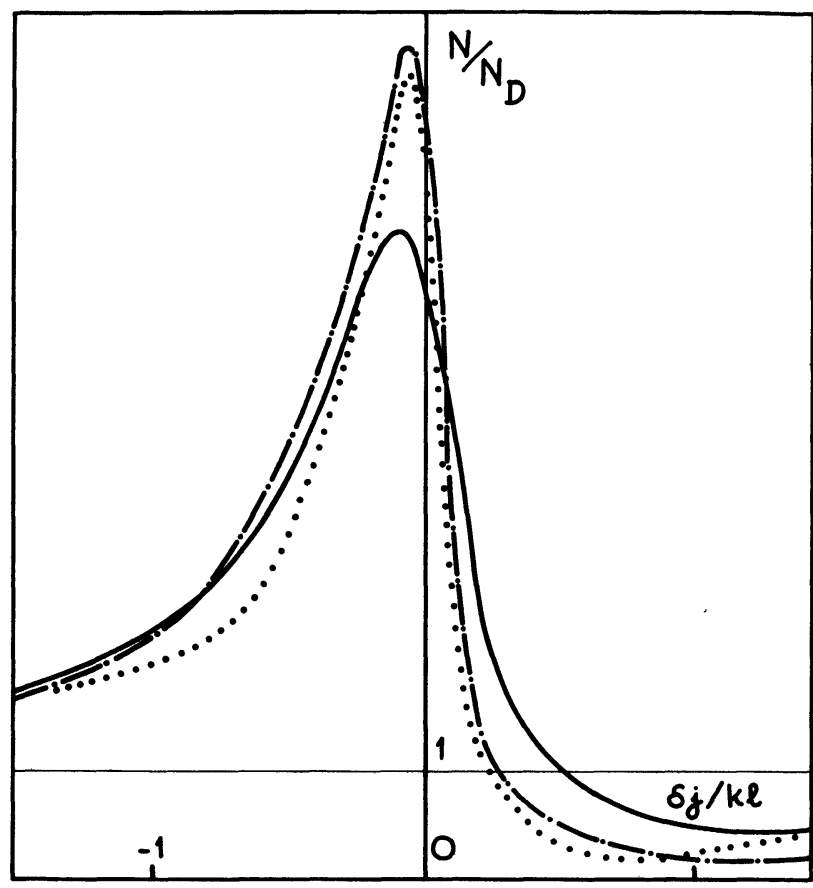

Fig. 2. - Influence of the pulse shape. $\theta=4 \pi, A_{M}^{2}=0.1$. Continuous excitation;.$--a) ; \ldots . b$ ).

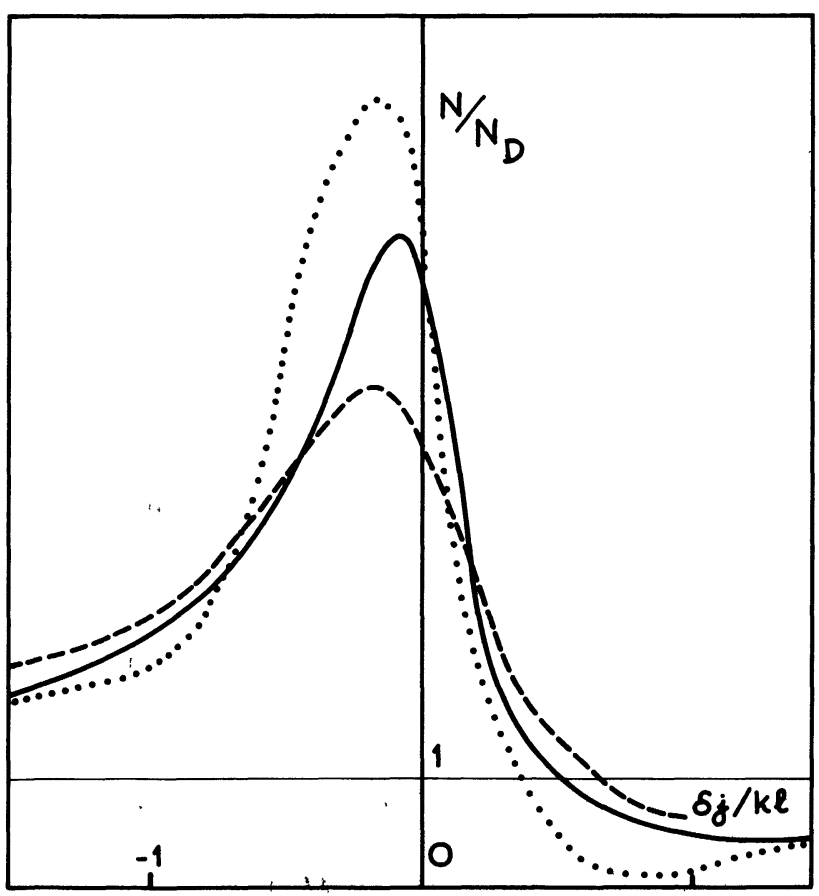

Fig. 3. - Influence of the pulse shape. $\theta=\pi, A_{\mathrm{M}}^{\dot{2}}=0.1$. Continuous excitation $; \ldots b$ ) ; - - - c).

For example, in the case considered $(n=3, p=1)$, in spite of the fact that the corresponding pulses are rather different, $x_{\mathrm{a}}^{2}$ and $x_{\mathrm{b}}^{2}$ are very close $(\sim 0.82)$ and the corresponding resonance profiles are quite similar (see Fig. 2). Moreover, one can again define a limit value of $\theta, \theta_{\mathrm{s}}$, in such a way that, for $\theta>\theta_{\mathrm{s}}$, the resonance profile is no longer dependent on $\theta$. For a given value of $A_{\mathrm{M}}^{2}, \theta_{\mathrm{s}}$ increases when $x^{2}$ decreases.

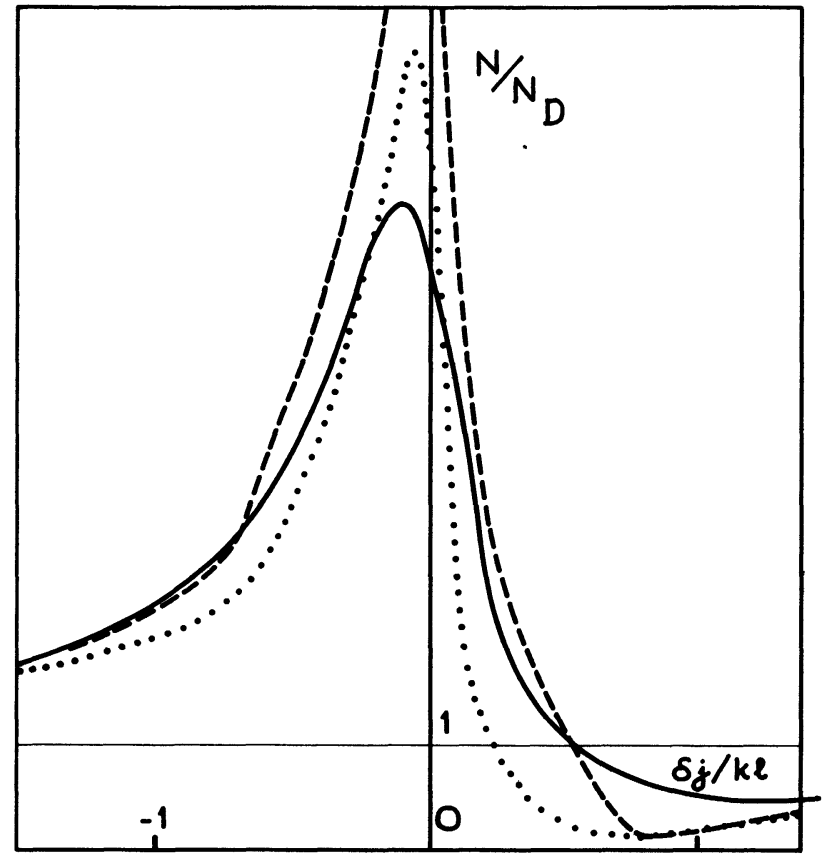

FIG. 4. - Influence of the pulse shape. $\theta=4 \pi, A_{\mathrm{M}}^{2}=0.1$. Continuous excitation ; . . b ) ; . . - c).

In any case, if we again try to characterize the spectral response function by an effective value of $A$, this quantity, $\hat{A}$, increases when $\theta$ decreases, as for a square pulse. Now, if we consider two pulses 1 and 2 with $x_{1}^{2}<x_{2}^{2}$, it appears that (for a given value of $A_{\mathrm{M}}$ )

$$
\begin{array}{ll}
\hat{A}_{1}^{2}>\hat{A}_{2}^{2} & \text { for small values of } \theta: \theta<\theta_{\mathrm{s} 1}, \theta_{\mathrm{s} 2}, \\
\hat{A}_{1}^{2}<\hat{A}_{2}^{2} & \text { for large values of } \theta: \theta>\theta_{\mathrm{s} 1}, \theta_{\mathrm{s} 2} .
\end{array}
$$

The results given in figures 3 and 4 are in qualitative agreement with these general rules since $x_{\mathrm{c}}^{2}=0.69$ is smaller than $x_{b}^{2}=0.83$, itself smaller than one, which is the value of $x^{2}$ for a square pulse. As a concluding remark on this point, it must be emphasized that the respective influences of the pulse area and of the pulse shape are rather intricate, and therefore, if these characteristics are not experimentally known, a relevant interpretation of resonance profiles in resonant multiphoton ionization is impossible in most cases.

Although time-dependent phases appear in the general formalism described here, we have considered in particular examples field amplitude variations only. Therefore, we have not introduced temporal coherence properties of the field, but it must be noted that these properties can play an important role in resonant multiphoton ionization as has been shown in recent papers [7]. Finally, it is clear that a knowledge of all the characteristics of excitation (pulse duration, pulse shape, temporal coherence) is essential to interpret in detail resonant multiphoton ionization processes. The formalism that we present here leads to a parametrization of resonance profiles which allows one to take into account all the field characteristics and, thus, to extract reliable atomic parameters. 


\section{References}

[1] See for example : Bakos, J. S., Adv. Electron. Electron Phys. [5] Beers, L. B. and Armstrong, L., Jr, Phys. Rev. A 12 (1975) 36 (1974) 57.

[5] Beers, L. B. and Armstrong, L., Jr, Phys. Rev. A 12 (1975)
2447.

[6] See for example : Allen, L. and Heberly, J. H., Optical Resonance and Two-level atoms (Wiley-Interscience, New York) 1975.

A 7 (1973) 2069.

Lambropoulos, P., Phys. Rev. A 9 (1974) 1992

[3] Feneullle, S. and Armstrong, L., Jr, J. Physique Lett. 36 (1975) L-235.

[4] Armstrong, L., Jr, Beers, L. B. and Feneuille, S., Phys. Rev. A 12 (1975) 1903.

[7] Mostowski, J., Phys. Lett. 56A (1976) 87.

Armstrong, L., Jr and Lambropoulos, P., Phys. Rev. Lett. 36 (1976) 952. 